\title{
Combined preventive/reactive congestion control in LAN/MAN interconnection networks
}

\author{
B.J. Lee and J.W. Mark \\ Department of Electrical and Computer Engineering \\ University of Waterloo \\ Waterloo, Ontario, Canada \\ Tel: (519) 888-4567, ext. 2532 \\ Fax: (519) 476-3077 \\ bjlee@bbcr.uwaterloo.ca \\ jwmark@bbcr.uwaterloo.ca
}

\begin{abstract}
This paper proposes and investigates the performance of a combined preventive/reactive congestion control approach for high-speed LAN/MAN interconnection networks. The interconnection of low-speed LANs via a high-speed MAN faces a speed-mismatch. As the primary control component, the preventive rate control mechanism is designed to cope with the speed-mismatch problem by throttling the long-term average input traffic injected into the backbone network under a large propagation delay constraint. The reactive feedback control component complements the preventive rate control to enhance the overall congestion control performance. Simulation results demonstrate the effectiveness of the proposed congestion control approach under different traffic conditions.
\end{abstract}

\section{Keywords}

LAN, MAN, interconnection, networks, preventive, reactive, congestion control

\section{INTRODUCTION}

One of the major applications envisaged for a high-speed MAN (Metropolitan Area Network) is the interconnection of geographically distributed LANs (Local Area Networks). For the last two decades, LANs for the support of communications amongst users within a small geographical area have proliferated. Such a small geographical coverage can now further be extended by interconnecting remotely located LANs via a high-speed backbone MAN. 
Major technical issues encountered in network interconnection include level of interconnection, addressing, routing, segmentation/reassembly and congestion control [Sunshine (1990)]. In particular, congestion control in LAN/MAN interconnection is a challenging problem because of the following conditions.

(1) The channel speeds of the backbone MAN and the attached LANs are mismatched.

(2) The backbone MAN exhibits a large propagation delay-bandwidth product and the LANs are geographically dispersed.

(3) Both the backbone MAN and the LANs typically support best-effort connectionless service.

(4) The LAN traffic exhibits unpredictable variabilities at all time scales [Fowler and Leland (1991), Leland and Wilson (1991)].

Congestion occurs when the aggregate demand on a resource (e.g., bandwidth of a link, buffer space, processing capacity at a bottleneck node) exceeds the available capacity [Jain (1990)], i.e.,

\section{$\sum$ Demand $>$ Available Resources.}

In a LAN/MAN interconnection network, condition (1) above brings out the intrinsic vulnerability to congestion occurrence due to such demand-capacity imbalance, especially for the traffic flow from the high-speed backbone MAN to the low-speed LAN. Condition (2) renders the use of purely reactive control ineffective. Conditions (3) and (4) also make it difficult to use prediction for preventive congestion control.

Most of the previous studies in this area are based on an overly simplifying assumption that the roundtrip propagation delay of the backbone MAN is negligible [Towsley, Fdida and Santoso (1992), Wong and Schwartz (1989), Inai and Ohtsuki (1992)]. Such an assumption implies that the overall network state information is available instantaneously.

This paper proposes a congestion control approach which combines preventive rate and reactive feedback control mechanisms. The preventive rate control component limits the long-term average input traffic destined for a particular destination LAN via the backbone MAN to cope with conditions (1) and (2) mentioned above. The reactive control component is complementary in nature and aims at controlling the short-term transient congestion due to conditions (3) and (4).

The following lists the desirable features in implementing the proposed congestion control strategy:

- essentially preventive rather than reactive

- distributed and adaptive

- robust with respect to different traffic characteristics

- fair in resource allocation

The LAN/MAN interconnection network model considered in this paper, along with a typical example congestion scenario, is briefly described in section 2 . Section 3 presents the overall framework and the implementation algorithms of the proposed congestion control approach. The effectiveness of the proposed control approach is investigated 


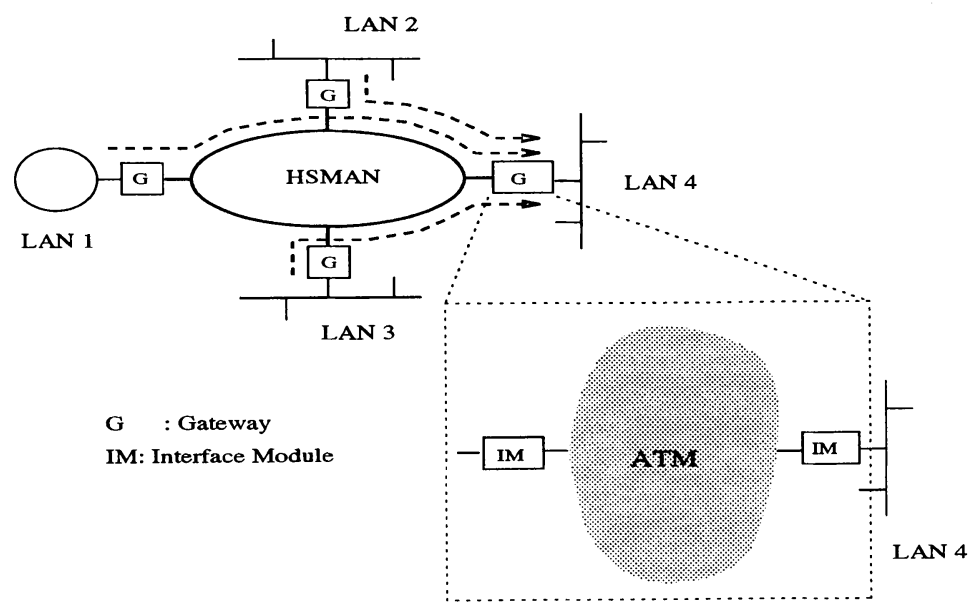

Figure 1 LAN/MAN interconnection network model.

in section 4 by means of computer simulation. Various performance aspects related to different implementation strategies of the reactive feedback control component are also discussed in section 4 . Concluding remarks are given in section 5 .

\section{LAN/MAN INTERCONNECTION}

\subsection{Network interconnection model}

The network interconnection model considered in this paper, where a number of low-speed LANs (e.g., Ethernet, IEEE 802.5 token ring, etc.) are interconnected via a high-speed backbone MAN, is depicted in Figure 1. We use the term input gateway to denote the gateway which handles traffic flowing from a source LAN to the backbone, and output gateway to denote the gateway which handles the flow of traffic from the backbone to the destination LAN. Figure 1 also shows a possible extension of the interconnection model [Gerla, Tai and Gallasi (1992)], where either the source or destination LAN is connected to the backbone MAN through an ATM (Asynchronous Transfer Mode) network.

A possible congestion scenario is depicted by the dashed lines in Figure 1, where interLAN traffic is being simultaneously transmitted to a common destination LAN. Because of the channel speed mismatch (i.e., the channel capacity of the backbone is much higher than that of the outgoing link to the destination LAN), the output gateway or Interface Module (IM) can easily be overloaded. Furthermore, because of the connectionless datagram service supported both in the LAN and the backbone MAN, and the geographical distribution of the LANs, prediction of such overload is very difficult, if not impossible. Once the output gateway (or the IM on the backbone MAN side) becomes overloaded, the lost traffic and its future retransmissions will further prevent other inter-LAN or intra-backbone MAN traffic from utilizing the backbone. 


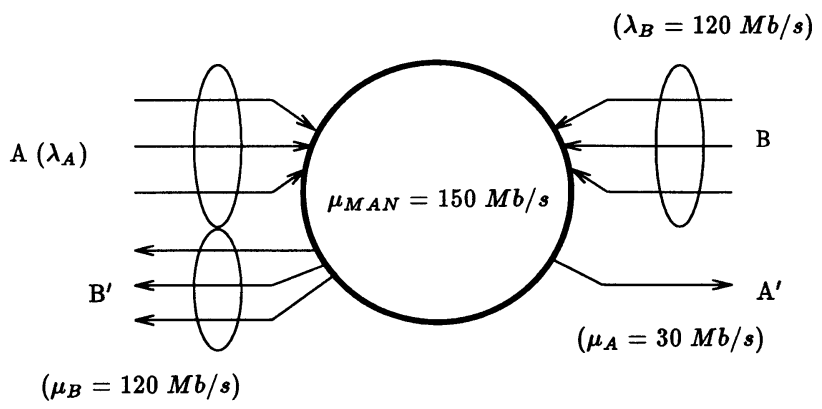

Figure 2 An example of uncontrolled congestion scenario.

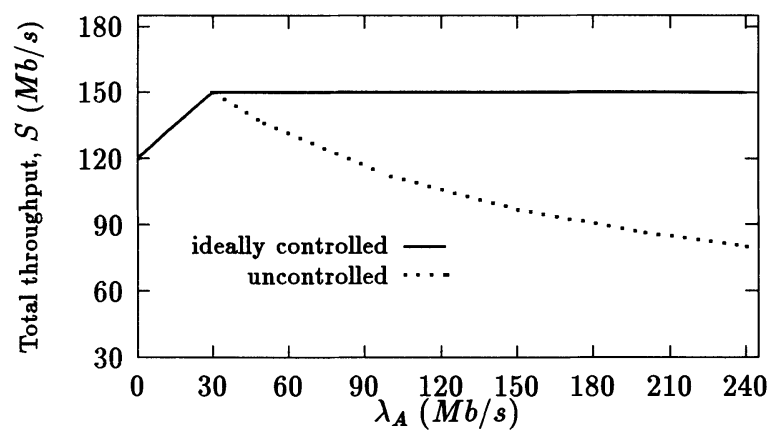

Figure 3 Throughput degradation due to uncontrolled congestion.

\subsection{Example congestion scenario}

Figure 2 illustrates an example congestion problem, where two groups of source-destination LAN connections, $\left(A, A^{\prime}\right)$ and $\left(B, B^{\prime}\right)$, are shown. Connection pair $\left(B, B^{\prime}\right)$ may include multiple source and destination LANs which utilize $80 \%$ of the backbone channel capacity. In connection $\left(A, A^{\prime}\right)$, multiple number of source LANs are simultaneously transmitting to a common destination LAN. The service capacity of $\mathrm{A}^{\prime}$ and $\mathrm{B}^{\prime}$ are given as 30 and 120 $M b / s$, respectively, while that of the backbone MAN is assumed to be $150 \mathrm{Mb} / \mathrm{s}$.

Assume that, if the total input traffic to the backbone exceeds $150 \mathrm{Mb} / \mathrm{s}$, the backbone MAC (Media Access Control) protocol allocates the channel capacity to each connection proportional to the amount of respective input traffic, $\lambda_{A}$ and $\lambda_{B}$. Then, if the amount of input from $\mathrm{B}$ is fixed at $120 \mathrm{Mb} / \mathrm{s}$, the total throughput at the destination LANs 
$\left(\mathrm{A}^{\prime}\right.$ and $\left.\mathrm{B}^{\prime}\right)$ can be expressed as a function of $\lambda_{A}$,

$$
S= \begin{cases}120+\lambda_{A}(M b / s), & \text { for } \lambda_{A} \leq 30 \\ 150\left(\frac{120}{\lambda_{A}+120}\right)+30(M b / s), & \text { for } \lambda_{A}>30\end{cases}
$$

and is plotted in Figure 3.

In the above example, the total effective throughput of the inter-LAN traffic $(A \rightarrow$ $\mathrm{A}^{\prime}$ and $\mathrm{B} \rightarrow \mathrm{B}^{\prime}$ ) increases until $\lambda_{A}$ reaches $\mu_{A}$, and then decreases thereafter. This phenomenon is due to excessive transmissions from the source LANs (A) exceeding the service capacity of the bottleneck $\left(A^{\prime}\right)$ and thus blocking other inter-LAN traffic $(B \rightarrow$ $\left.B^{\prime}\right)$ from entering the backbone. With a proper control mechanism regulating the interLAN traffic flow $\left(A \rightarrow A^{\prime}\right)$ not to exceed the service capacity of $A^{\prime}$, the total effective throughput could be maintained at its maximum (shown as ideally controlled in Figure 3).

\subsection{Level of interconnection}

Another major issue regarding the gateway design is at what level in the protocol stack to interconnect networks. Although alternatives exist from the lowest (physical) layer to the highest (application) layer [Sunshine (1990)], our practical interest lies in the data link layer interconnection for the following reason.

The class of high-speed MANs considered in this paper is basically an extended LAN stretched over a larger geographical area, e.g., CTRA [Mark, Lee and Mark (1994)], DQDB [IEEE 802.6 (1990)], CRMA [Nassehi (1990)], and ATMR [ISO/IEC (1992)]. Therefore, what distinguishes the backbone MAN from the LAN is the MAC protocol which is designed to maximize the channel utilization regardless of the network size and speed. Consequently, the minimum required functionality for our LAN/MAN interconnection is a relay mechanism at the boundary between two different MAC protocols. Such devices are called $M A C$ bridges, which interconnect networks at the MAC layer sometimes at the LLC (Logical Link Control) layer [Gerla and Kleinrock (1988)]. Their main attribute is transparency and they do not interfere with the end-user's protocol layers (i.e., network and above).

However, the MAC bridges are not equipped to deal with congestion occurring at the network boundaries due to the speed mismatch and/or the load unbalancing as pointed out earlier, although the MAC protocol itself can effectively control congestion in a single LAN or MAN, e.g., round-robin access in token rings and buses, binary back-off scheme in CSMA-CD (Carrier Sense Multiple Access with Collision Detection), etc. [Gerla and Kleinrock (1988)]. Therefore, extra functionality is needed on top of the MAC relay mechanism at the MAC bridges. In this paper, the LAN/MAN interconnection is assumed to be realized at the MAC layer and the proposed congestion control mechanisms are implemented on top of the MAC relay function (see Figure 4).

\subsection{Layered architecture}

Figure 4 depicts a layered architecture of our LAN/MAN interconnection network model. The extra layer on top of the MAC relay function in the gateway represents a superimposed congestion control functionality. Data exchange between an end-user $A$ on a source 
user A

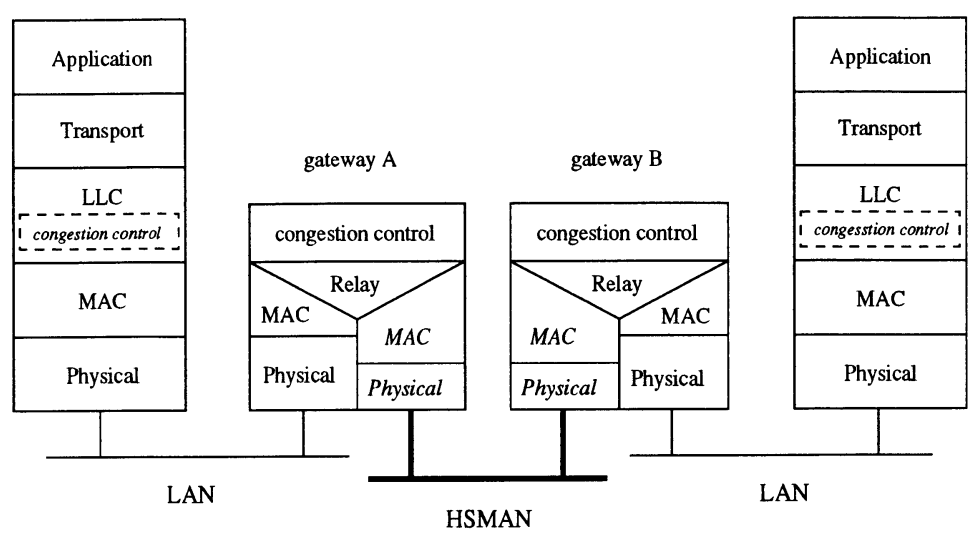

Figure 4 Layered architecture of a LAN/MAN interconnection network model and a gateway implementation.

LAN and an end-user $B$ on a destination LAN through a backbone MAN proceeds as follows. Assume that these two users share an identical protocol stack. The data to be sent by the application process of user $A$ is first encapsulated with the transport protocol header and then with the LLC header. Subsequently, the LLC PDU (Protocol Data Unit) is again encapsulated with the MAC protocol header of the LAN and sent to input gateway $A$. Gateway $A$ wraps it with the MAC protocol header of the backbone MAN and sends it to output gateway $B$. Then, gateway $B$ strips off the MAN header and forwards the MAC PDU of the LAN to user $B$.

If the two end-users do not share an identical protocol stack below the LLC level (e.g., between an Ethernet user and an IEEE 802.5 token ring user), then the gateways have to perform MAC protocol conversion as a relay function. In either case, the intermediate gateways exercise control on the flow of the converted MAC PDUs of the backbone MAN. Additional feedback control between the end-user and the input gateway may also be desirable, as shown by the dashed box embedded between the LLC and the MAC layers in the end-user protocol stack.

\section{COMBINED PREVENTIVE/REACTIVE CONTROL}

This section presents the overall congestion control framework and describes four different preventive/reactive components for enforcing congestion control.

\subsection{Overall control framework}

The overall control framework, employing a combination of preventive and reactive control strategies, consists of four control components, as indicated in Figure 5. The preventive control component $\mathrm{C} 1$ is the primary control function which throttles the inter-LAN 


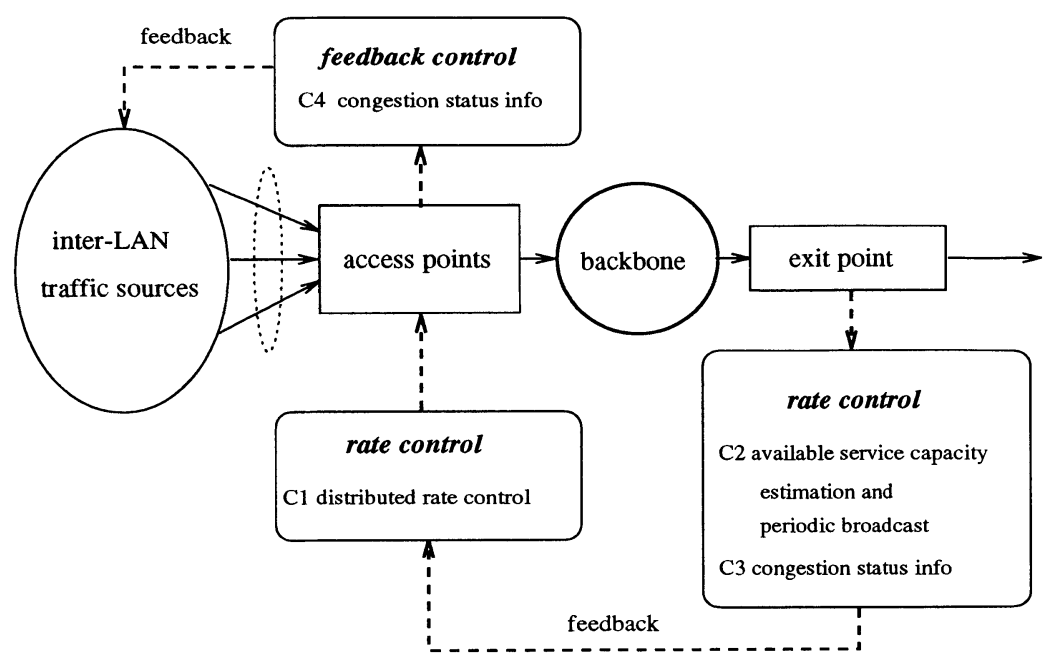

Figure 5 Overall preventive/reactive congestion control framework.

traffic at the access points (input gateways) not to exceed the estimated service capacity of the bottleneck exit point (output gateway). Control component C2 estimates and broadcasts the long-term service capacity of the bottleneck output gateway (or the Interface Module, see Figure 1). In this paper, it is assumed that the estimated service capacity is known a priori in terms of mean service rate in $M b / s$. Control components $\mathrm{C} 1$ and $\mathrm{C} 2$ together can be viewed as an emulation of bandwidth negotiation/reservation at connection setup, and preventive bandwidth enforcement in an ATM network.

The reactive feedback control component $\mathrm{C} 3$ provides fine-tuning of the preventive control C1 by dynamically adjusting the control parameter(s) of C1. Assuming negligible feedback propagation delay between the end users in the source LAN and the corresponding input gateway, the reactive control component $\mathrm{C} 4$ can also be highly effective in maintaining a low input gateway buffer size and in throttling the source traffic. In the sequel, we are only concerned with control strategies $\mathrm{C} 1$ and $\mathrm{C} 3$, leaving $\mathrm{C} 2$ and $\mathrm{C} 4$ for future consideration.

\subsection{Preventive rate control $\mathrm{C1}$}

The preventive rate control $\mathrm{C} 1$ is to throttle the long-term average input traffic load, $\lambda^{c}$, destined for the output gateway not to exceed the estimated mean service rate, $\mu_{o}$, of the output gateway, i.e.,

$$
\lambda^{c}=\sum_{i} \lambda_{i}^{c} \leq \mu_{o}
$$

where $\lambda_{i}^{c}$ denotes the controlled average input rate from the $i$ th input gateway. It is assumed that $\mu_{o}$ is estimated at the output gateway by monitoring the intra-LAN traffic of the destination LAN. In the case of an IM connecting the backbone MAN to the 


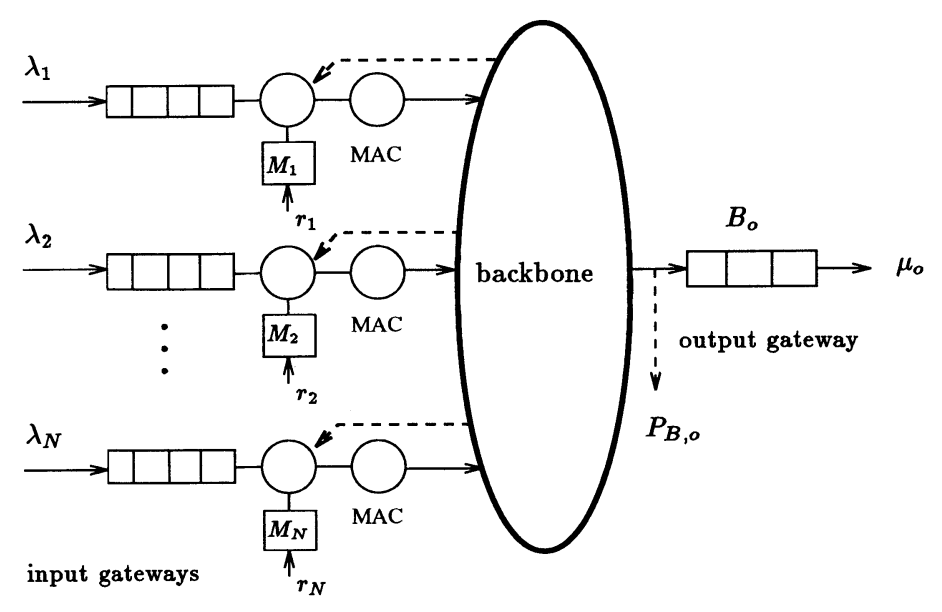

Figure 6 An inter-LAN traffic flow controlled by distributed LB controller.

ATM network (see Figure 1), the available mean service rate may also depend on how the best-effort connectionless data service is supported through the connection-oriented ATM network [Gerla, Tai and Gallasi (1992), Crocetti, Gallasi and Gerla (1991), Newman (1993)].

If all the input gateways are clustered together at the same location, then a single rate control mechanism (e.g., leaky bucket rate controller [Sidi, Liu, Cidon and Gopal (1989)]) can be employed to regulate the aggregate traffic flow. However, in our case, the input gateways are geographically distributed and thus some form of distributed coordination among the input gateways is necessary to emulate such a single global rate control mechanism. A distributed leaky-bucket (LB) rate control algorithm is proposed in [Lee and Mark (1994)], which makes use of the broadcast shared-medium topology of the backbone MAN to realize such a coordination in a distributed manner. The algorithm is briefly described in the next subsection.

\subsubsection{Distributed LB rate control algorithm}

Figure 6 shows $N$ traffic sources destined to a common output gateway via a backbone network. Also shown are the distributed LB rate controllers. An implementation of the distributed LB rate control algorithm at the $i$ th input gateway is shown in Figure 7 . The $i$ th local LB controller monitors the network-wide traffic condition (maybe somewhat out-dated and/or incomplete) by observing the transit traffic passing by in the channel and uses it to regulate the local input traffic, $\lambda_{i}$, from the corresponding source LAN. As 


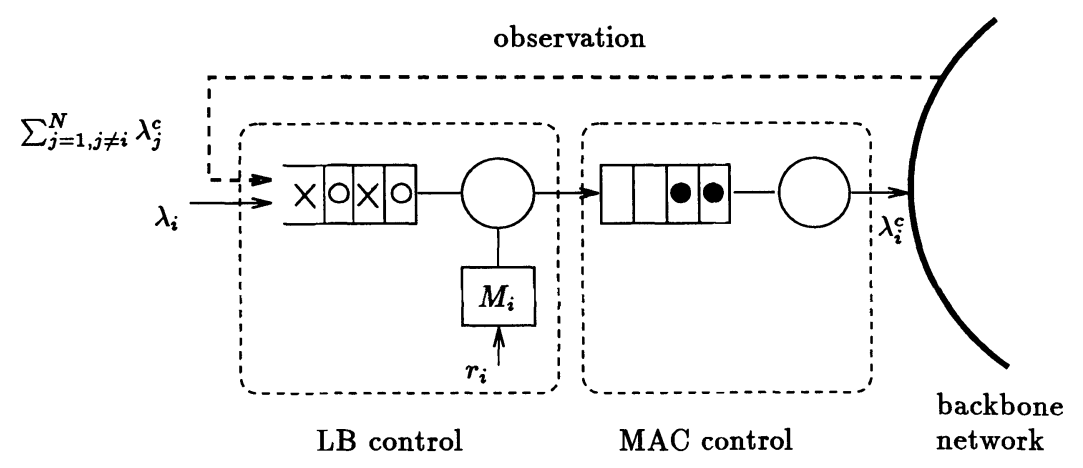

Figure 7 Implementation of distributed LB rate control algorithm.

a result, the controlled traffic from the $i$ th input gateway, $\lambda_{i}^{c}$, can be expressed as

$$
\lambda_{i}^{c}=f\left(\sum_{j=1, j \neq i}^{N} \lambda_{j}^{c}, \lambda_{i}\right),
$$

where $f(\cdot)$ denotes the local LB rate control function with control parameters, $r_{i}$ and $M_{i}$ (representing the LB permit generation rate and the permit pool size, respectively).

In other words, the traffic generated from the local LB controller is regulated by the network-wide traffic and, in turn, the network-wide traffic is again moderated by the local traffic generated by the $i$ th input gateway. In this paper, the local permit generation rates are assumed to be identical for all the input gateways, i.e., $r_{i}=r \forall i$, which will limit the long-term aggregate average input rate to the output gateway not to exceed $r$. Hereafter, $r$ is referred to as the global permit generation rate.

The algorithm works as follows:

- Observation of a transit packet (depicted as a cross mark in Figure 7) causes a LB permit to be consumed, which is to account for the iraffic already injected into the backbone network from the other input gateways.

- Locally generated packets (depicted as empty circles) and the observation marks of the transit traffic are enqueued in the LB buffer and consume LB permits in their arriving order.

- If a usable permit is captured by a locally generated packet, then the packet joins the MAC buffer (represented by solid circles) and becomes ready for transmission whenever the backbone MAC allows.

Note that, in Figure 7, the LB rate control function is separated from the backbone MAC control and a FIFO (first-in-first-out) queueing discipline is adopted for the aggregated observed and locally generated traffic. The separation of the LB rate control and the backbone MAC functions conforms to the proposed layered architecture of gateway 
implementation depicted in Figure 4. The FIFO discipline is an effort to provide a fair share of usable permits to the locally generated traffic, especially when the transit traffic is heavy.

\subsection{Reactive feedback control C3}

The reactive feedback control component C3 complements the preventive control function $\mathrm{C} 1$ to provide additional performance enhancement under various traffic conditions. Recall that the preventive rate control $\mathrm{C} 1$ is only concerned with matching the long-term average input and service rates as prescribed in Eqn. (1). In order to meet the more specific performance requirements (e.g., a certain amount of throughput is required with a given loss probability constraint at the output gateway), the control parameters of $\mathrm{C} 1$ may need to be dynamically adjusted by the reactive feedback control.

The reactive feedback control C3 consists of two parts: 1) congestion detection mechanism at the bottleneck output gateway and 2) adjustment policy of the C1 control parameters at the input gateways based on the congestion status information.

\subsubsection{Congestion detection}

Congestion at the output gateway is detected by monitoring the average output queue length variation against a preset threshold $T h$. By using the average instead of instantaneous queue length variation, unnecessary control signal generation can be avoided. The average queue length is estimated recursively using an exponentially weighted moving average (EWMA):

$$
\bar{q}_{t}=\left(1-w_{q}\right) \bar{q}_{t-1}+w_{q} q_{t},
$$

where $\bar{q}_{t}$ and $q_{t}$ are respectively the average and instantaneous queue length observed by a packet arrival at time $t . \bar{q}_{t-1}$ is the average queue length calculated at the time of the last packet arrival prior to $t$.

The weight, $w_{q}$, determines the time constant of the low-pass filtering process and thus can be manipulated to control the magnitude and duration of the short-term transient congestion as desired. Note that, if $w_{q}=1$, then $\bar{q}_{t}=q_{t}$, and the congestion detection is based on the instantaneous queue length variation.

When the average queue length, $\bar{q}_{t}$, increases beyond the preset threshold, $T h$, i.e., $\bar{q}_{t-1}<T h<\bar{q}_{t}$, a congestion indication is broadcasted. On the other hand, when $\bar{q}_{t-1}>T h>\bar{q}_{t}$, a different control signal indicating such condition is broadcasted.

\subsubsection{Dynamic rate adjustment policy}

When the congestion indication is received at the input gateways, the control parameters of the LB rate control mechanism (e.g., permit generation rate and/or pool size) can be adjusted accordingly. In this paper, only the global permit generation rate, $r$, is adjusted ${ }^{1}$ and two rate adjustment policies are investigated: 1) step-wise and 2) on-off increment/decrement policies.

\footnotetext{
${ }^{1}$ Although the permit pool size, $M_{i}$, may also be adjusted, simulation results indicate that variations in $M_{i}$ has little effect on the performance.
} 
1. Step-wise policy

- upon receiving the congestion indication: set $r \rightarrow r_{L}=(1-\Delta) r^{*}$

- otherwise: $\operatorname{set} r \rightarrow r_{H}=(1+\Delta) r^{*}$

- $\Delta$ is the increment/decrement step size

- $r^{*}\left(\leq \mu_{o}\right)$ is the target operating permit generation rate

- $\left(r_{L}+r_{H}\right) / 2=r^{*}$

2. On-off policy

- upon receiving the congestion indication: $r \rightarrow 0$

- otherwise: $r \rightarrow r_{p}>r^{*}$

The on-off increment/decrement policy is, in fact, a special case of the step-wise adjustment policy. As will be shown later, the on-off scheme is devised to better handle the bursty and correlated on-off traffic model, and as such, $r_{p}$ is set to match the peak input rate of the on-off traffic model.

\section{PERFORMANCE EVALUATION}

In this section, simulation results are presented to evaluate the effectiveness of the control components $\mathrm{C} 1$ and $\mathrm{C} 3$ under different traffic assumptions, e.g, Poisson and exponential on-off traffic. Two performance aspects are of particular interest: 1) the effectiveness of the distributed LB rate control algorithm in throttling the input traffic, and 2) the degree of performance enhancement attainable by the reactive control component C3.

\subsection{Backbone network architecture}

For simulation study, a particular backbone MAN architecture is used, namely the CTRA (Combined Token/Random Access) ring network [Mark, Lee and Mark (1994)]. The CTRA protocol combines a token access control with an add-on random access operation to maximize the channel utilization, and is designed for integrated services high-speed ring networks (see [Mark, Lee and Mark (1994)] for detailed description). In the CTRA backbone, a source removal policy is also used so that the complete channel traffic can be observed at each input gateway. Note that, if complete channel traffic information is not observable due to the topology and/or operational features of the backbone network (e.g., unidirectional bus topology as in DQDB and CRMA, destination removal as in ATMR, or slot reuse as in DQDB), then the permit consumption rule for the traffic arriving from the source LAN may further be adjusted in some statistical manner (see Figure 7).

\subsection{Traffic model}

Two different traffic models are considered: 1) Poisson and 2) exponentially distributed on-off traffic. In the Poisson traffic model, the inter-LAN traffic arrival process from a source LAN to the corresponding input gateway is assumed Poisson. To account for the 
Table 1 Parameter values used in the simulation study

\begin{tabular}{|c|c|}
\hline \multicolumn{2}{|c|}{ network parameters } \\
\hline$N=10$ & number of input gateways \\
\hline$\mu_{o}=10(M b / s)$ & mean service rate at the output gateway \\
\hline$\mu_{M A N}=150(M b / s)$ & backbone MAN speed \\
\hline$B_{I}=100$ & input gateway buffer size \\
\hline$B_{o}=30,60,100$ & output gateway buffer size \\
\hline $\begin{aligned} \alpha= & 30,300,500,1000 \\
& (40,400,666,1333 \mathrm{Km})\end{aligned}$ & normalized roundtrip propagation delay \\
\hline \multicolumn{2}{|c|}{ control parameters of $C 1$ and $C 3$} \\
\hline$r \leq \mu_{o}$ & global permit generation rate \\
\hline $\bar{M}=10$ & permit pool size \\
\hline$w_{q}=0.1$ & weighting constant in EWMA \\
\hline$T h=B_{o} / 2=30,50$ & threshold value for congestion detection \\
\hline$\Delta=0.2$ & step size for step-wise rate adjustment scheme \\
\hline \multicolumn{2}{|c|}{ on-off traffic model parameters } \\
\hline $\bar{T}=15(0.1 \mathrm{~ms})$ & fixed packet generation/service interval \\
\hline$\nu^{-1}=450(3 \mathrm{~ms})$ & input mean 'on' period \\
\hline $\begin{aligned} \beta^{-1}= & 1050,2550,5550,7050 \\
& (7,17,37,47 \mathrm{~ms})\end{aligned}$ & input mean 'off' period \\
\hline$\nu_{o}^{-1}=1500(10 \mathrm{~ms})$ & output mean 'on' period \\
\hline$\beta_{o}^{-1}=500(3.33 \mathrm{~ms})$ & output mean 'off' period \\
\hline
\end{tabular}

service time variations at the output gateway due to intra-LAN traffic fluctuations of the destination LAN, the service time of the inter-LAN traffic at the output gateway is also assumed to be exponentially distributed.

It is believed, however, that the LAN traffic and the inter-LAN traffic generated from a source LAN exhibit bursty characteristics [Leland and Wilson (1991), Jain (1990)]. We approximate such LAN traffic characteristics by modeling the inter-LAN traffic entering the input gateway and the service process at the output gateway as exponentially distributed on-off processes.

\subsection{Simulation model parameters}

The speed ratio between the backbone MAN and the source/destination LAN is assumed to be 15:1. $N+1$ gateways are equidistantly attached to the backbone, where $N$ input gateways are transmitting their packets to a common output gateway. The input and output gateway buffers are of a finite size and denoted as $B_{I}$ and $B_{o}$, respectively. The roundtrip propagation delay of the backbone ring normalized by packet transmission time is an important network parameter and is denoted by $\alpha$. The control parameters of all the distributed LB rate controllers are set identical, i.e., $r_{i}=r$ and $M_{i}=M \forall i$.

In the Poisson traffic model case, fixed-length packets are generated to the input gateway buffer at exponentially distributed time intervals with a mean arrival rate of $\lambda_{i}=\lambda \forall i$. The service time at the output gateway is also exponentially distributed with 


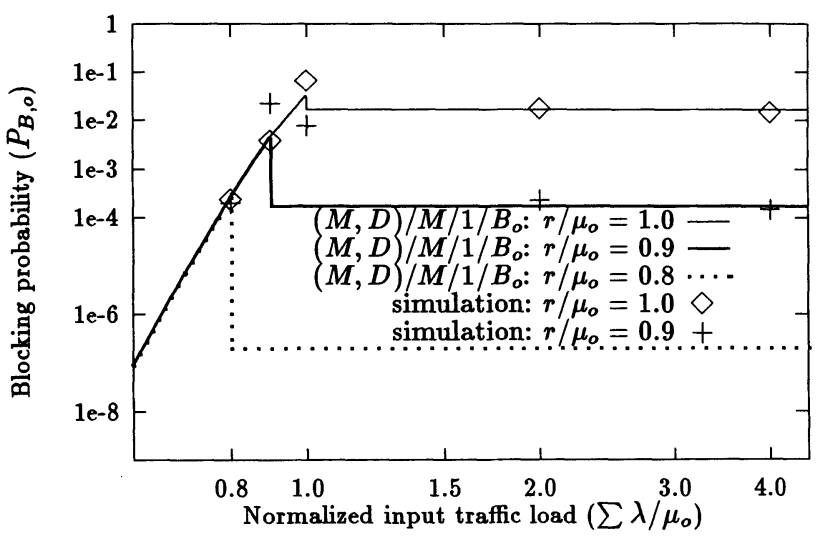

Figure 8 LB rate control: Poisson traffic, $\alpha=30, B_{o}=30$.

a mean service rate of $\mu_{o}$. On the other hand, in the on-off traffic model, fixed-length packets are generated at fixed time intervals during the 'on' period with the peak rate of $\gamma_{i}=\gamma \forall i$. The packet generation interval, $T$, corresponds to the time period required for the segmentation and conversion of the LAN packets into the fixed-length backbone MAC PDU (Protocol Data Unit). At the output gateway, the packet transmission is deterministic with a transmission time of $T$ during the service 'on' period, which is equivalent to the peak service rate of $\gamma$.

The exponentially distributed 'on' and 'off' periods at the input/output gateways are assumed independent with mean durations of $\nu_{i}^{-1}=\nu^{-1}$ and $\beta_{i}^{-1}=\beta^{-1} \forall i$, for the interLAN input processes, and $\nu_{o}^{-1}$ and $\beta_{o}^{-1}$ for the service process at the output gateway. Therefore, the mean input rate from a source LAN is denoted as $\lambda=\gamma\left(\frac{\beta}{\nu+\beta}\right)$, while $\mu_{o}=\gamma\left(\frac{\beta_{o}}{\nu_{o}+\beta_{o}}\right)$ represents the mean service rate at the output gateway. The simulation model parameters and the corresponding physical values are summarized in Table 1.

\subsection{Distributed LB rate control performance}

This section investigates the performance and the effect of parameter dimensioning of the distributed LB rate control algorithm. In Figure 8, the blocking probabilities, $P_{B, o}$, observed at the output gateway with a buffer size of $B_{o}=30$ are plotted for different settings of permit generation rate under various aggregate input traffic loads. The global permit generation rates are normalized by $\mu_{o}$. Similar results for $B_{o}=60$ are shown in Figure 9. Figure 10 also plots $P_{B, o}$ obtained for different output buffer sizes, $B_{o}$, with fixed global permit generation rate, $r=\mu_{o}$. Note that, in Figure 10, the setting for $r$ seems rather optimistic, i.e., the traffic intensity at the output gateway becomes unity $\left(\rho_{o} \triangleq r / \mu_{o}=1\right)$, which results in high blocking probabilities.

In Figures 8, 9 and 10, simple approximate analytical results for the Poisson traffic 


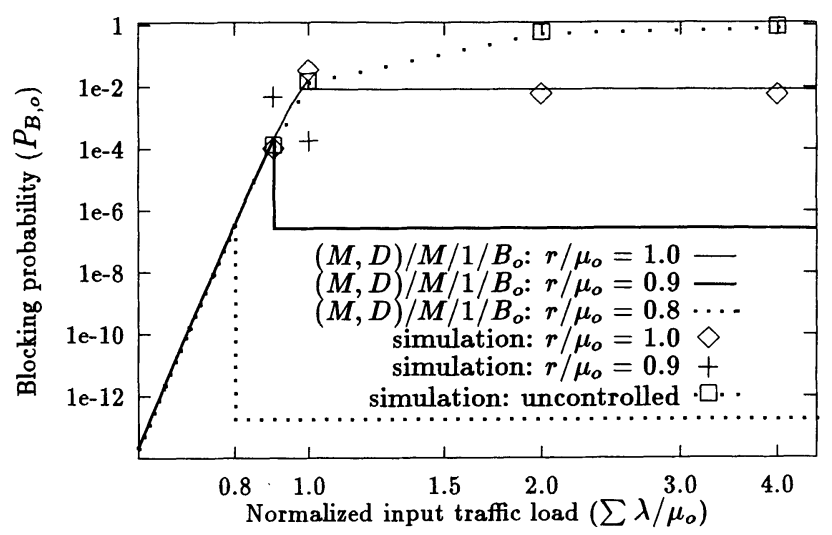

Figure 9 LB rate control: Poisson traffic, $\alpha=30, B_{o}=60$.

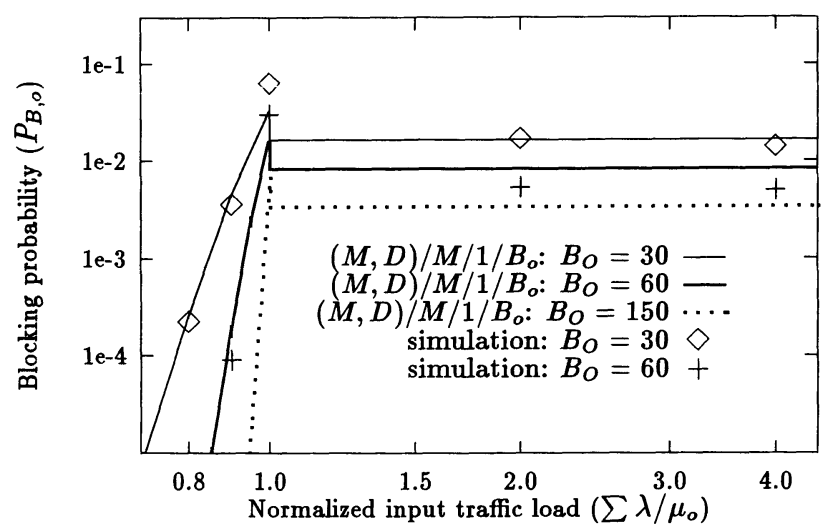

Figure 10 LB rate control: Poisson traffic, $\alpha=30, r=\mu_{o}$. 


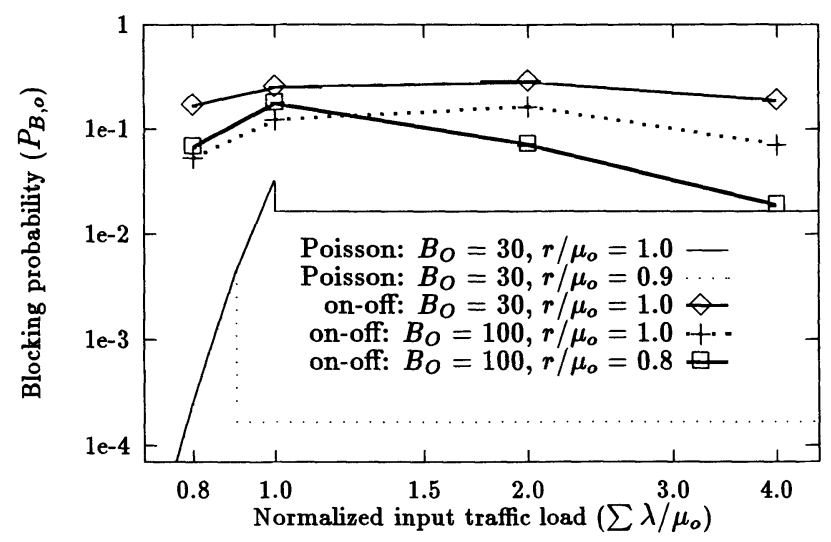

Figure 11 LB rate control: comparison of performance for Poisson and exponential on-off traffic, $\alpha=30$.

model are also shown against the simulation results. The approximate analytical results are obtained using a piecewise single server queueing model with a finite buffer size, i.e., $(M, D) / M / 1 / B_{o}$. For example, when the aggregate input traffic load offered to the backbone is less than the global permit generation rate, i.e., $\sum \lambda<r$, the $M / M / 1 / B_{o}$ model is used, while for $\sum \lambda>r$, we use the $D / M / 1 / B_{o}$ model.

Such a piecewise single server model is based on the conjecture that the distributed LB algorithm may also be approximated as a single global LB rate controller. Therefore, when $\sum \lambda>r$, the departure process from the LB rate control mechanism (i.e., the arrival process to the output gateway) is approximated as a deterministic process with a rate $r$. As a result, the queueing behaviour at the output gateway is approximated using the $D / M / 1 / B_{o}$ model. On the other hand, when $\sum \lambda<r$, assuming that the input Poisson process offered to the backbone is preserved through the LB control, the $M / M / 1 / B_{\text {o }}$ model is used.

It is observed that the simulation results closely match those of the analytical approximation in the Poisson traffic case, except when $\sum \lambda \approx r$, which proves the effectiveness of the distributed LB algorithm in emulating a single global LB controller. Although not shown in this paper, extensive simulation study also confirms such effect for various values of $r$. In other words, the distributed LB rate control algorithm effectively limits the aggregate input traffic rate not to exceed the mean service rate of the output gateway with $r \leq \mu_{o}$. Furthermore, note that under the Poisson traffic assumption, the blocking probabilities at the output gateway can significantly be mitigated by properly dimensioning $r$ and $B_{o}$, without severely degrading the throughput performance.

However, Figure 11 shows that, in the case of the exponential on-off traffic model, the blocking probability performance does not improve significantly by decreasing $r$ (e.g., from 1.0 to 0.8 ) or increasing the output buffer size, $B_{o}$ (e.g., from 30 to 100). Note 


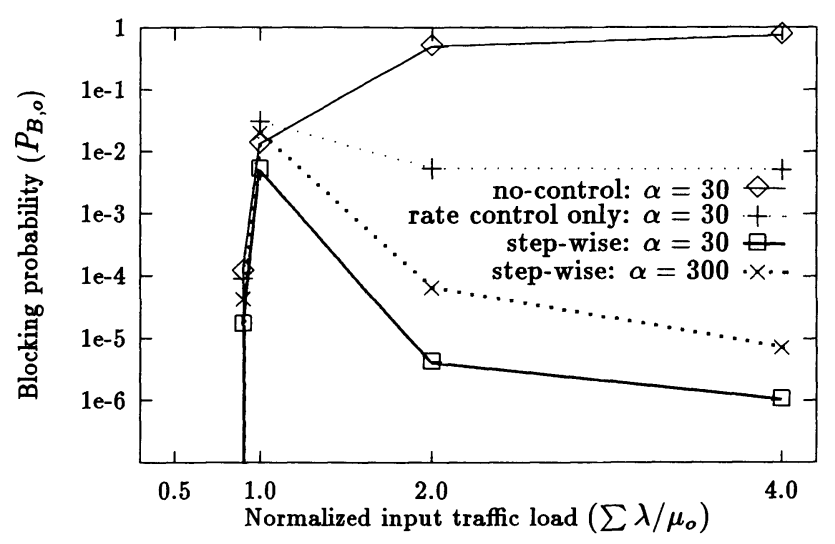

Figure 12 Poisson traffic: performance comparison of preventive rate control only vs. combined control, $B_{o}=60, T h=30, r=\mu_{o}$.

that the smaller value of $r$ results in lower throughput at the output gateway. The $(M, D) / M / 1 / B_{o}$ results from Figure 8 for the Poisson traffic case are also plotted for comparison purposes. In the next section, it will be shown that by combining a proper reactive feedback control function, the blocking and throughput performances can significantly be enhanced even for the on-off traffic model even with an optimistic setting of $r=\mu_{o}$.

\subsection{Combined control performance}

Figure 12 demonstrates the performance enhancement in terms of the blocking probability, $P_{B, o}$, achieved by combining the reactive feedback control function $\mathrm{C} 3$ with the preventive $L B$ rate control $C 1$. In the figure, we use the Poisson traffic model and the step-wise rate adjustment policy with a step size of $\Delta=0.2$ for the reactive control. Note that when the roundtrip propagation delay increases, i.e., from $\alpha=30$ to 300 , the performance also degrades, approaching that of the distributed LB rate control scheme without the reactive control C3.

In Figure 13, the blocking probabilities are compared for different parameter values for the exponential on-off traffic model. First of all, when $\alpha=30$ and the on-off rate adjustment policy is used for the reactive control $\mathrm{C} 3$, no packet blocking is observed at the output gateway with a buffer size of $B_{o}=100$ (see Table 2), which is a drastic performance improvement. In contrast, however, using the step-wise rate adjustment policy with a step size of $\Delta=0.2$ does not result in sizable performance improvement over the preventive rate control alone, i.e., without C3. As also shown earlier in Figure 11 , the smaller setting of $r / \mu_{o}=0.8$ (thus reducing the input traffic intensity, $\rho_{o}$, to the output gateway and the resulting throughput) also shows no significant improvement. As 


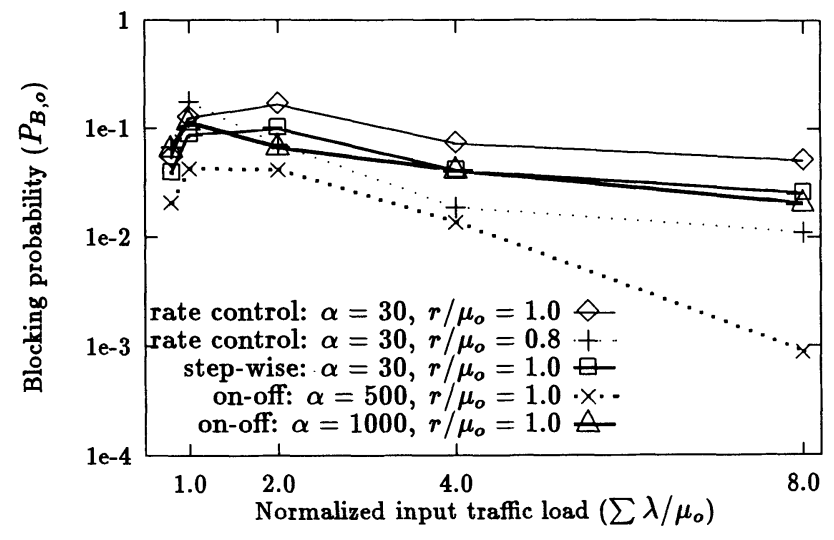

Figure 13 Exponential on-off traffic: performance comparison of various control schemes, $B_{o}=100, T h=50$.

in the Poisson traffic case, when $\alpha$ increases, the blocking probability performance also approaches that of the preventive rate control $\mathrm{C} 1$.

More detailed blocking and throughput performance results are tabulated in Table 2. In the table, the first column represents the total aggregate input traffic load, $\sum \lambda$, offered to the backbone MAN. $S_{b}$ and $S_{o}$ denote the throughput obtained in the backbone and at the output gateway, respectively. The blocking probabilities observed at the input and output gateways are shown as $P_{B, I}$ and $P_{B, o}$, respectively. Finally, $S_{b, L}\left(\triangleq S_{b}-S_{o}\right)$ quantifies the amount of traffic unnecessarily occupying the backbone channel due to blocking at the output gateway. Note that when $\alpha=30$ and the on-off rate adjustment policy is used for the reactive control, we obtain the largest throughput and zero blocking probability. The preventive rate control without reactive control component $\mathrm{C} 3$ shows the worst performance. Note also that, as in the case of the Poisson traffic model, the distributed LB rate control algorithm succeeds in throttling the aggregate input rate not to exceed the output service rate, $\mu_{o}=10 \mathrm{Mb} / \mathrm{s}$.

Dynamics of the queue length variation at the output gateway for the on-off traffic model has also been extensively observed through simulation to investigate the effects of different reactive control policies and propagation delays. Confirming the expectation, the queue length fluctuations have been rather drastic under the distributed LB rate control and the step-wise rate adjustment policy failed to reduce it. On the other hand, the on-off rate adjustment policy was found to be successful in confining the queue length fluctuations within a small range around the threshold values resulting in higher throughput and negligible blocking probabilities (see Table 2). However, as the roundtrip propagation delay increased, the magnitude of fluctuations increased again. The effects of different settings of EWMA weights, $w_{q}$, and the thresholds, $T h$, under various traffic conditions need further investigations. 
Table 2 Exponential on-off traffic: blocking and throughput performances of various control schemes, $B_{o}=100, T h=50, r=\mu_{o}=10 \mathrm{Mb} / \mathrm{s}$

\begin{tabular}{|c|c|c|c|c|c|}
\hline$\sum \lambda(M b / s)$ & $P_{B, I}$ & $S_{b}(M b / s)$ & $P_{B, o}$ & $S_{o}(M b / s)$ & $S_{b, L}(M b / s)$ \\
\hline \multicolumn{6}{|c|}{ preventive rate control only, $\alpha=30$} \\
\hline 8 & 0.0086 & 7.93 & 0.0530 & 7.51 & 0.42 \\
\hline 10 & 0.0428 & 8.61 & $\underline{0.1231}$ & $\underline{7.55}$ & 1.06 \\
\hline 20 & 0.4916 & 10.16 & $\underline{0.1648}$ & $\underline{8.49}$ & 1.67 \\
\hline 40 & 0.7473 & 10.10 & 0.0710 & 9.38 & 0.71 \\
\hline 80 & 0.8742 & 10.05 & 0.0498 & 9.55 & 0.50 \\
\hline \multicolumn{6}{|c|}{ combined, step-wise rate adjustment, $\alpha=30$} \\
\hline 8 & 0.0105 & 7.91 & 0.0381 & 7.61 & 0.30 \\
\hline 10 & 0.0490 & 9.50 & $\underline{0.0859}$ & $\underline{8.69}$ & 0.81 \\
\hline 20 & 0.4942 & 10.11 & $\underline{0.0992}$ & $\underline{9.11}$ & 1.00 \\
\hline 40 & 0.7464 & 10.14 & 0.0402 & 9.73 & 0.40 \\
\hline 80 & 0.8714 & 10.28 & 0.0250 & 10.02 & 0.25 \\
\hline \multicolumn{6}{|c|}{ combined, on/off rate adjustment, $\alpha=30$} \\
\hline 8 & 0.0246 & 7.80 & 0.0000 & 7.80 & 0.00 \\
\hline 10 & 0.0991 & 9.00 & $\underline{0.0000}$ & $\underline{9.00}$ & 0.00 \\
\hline 20 & 0.5243 & 9.51 & $\underline{0.0000}$ & $\underline{9.51}$ & 0.00 \\
\hline 40 & 0.7547 & 9.80 & 0.0000 & 9.80 & 0.00 \\
\hline 80 & 0.8738 & 10.09 & 0.0000 & 10.09 & 0.00 \\
\hline \multicolumn{6}{|c|}{ combined, on/off rate adjustment, $\alpha=500$} \\
\hline 8 & 0.0257 & 7.79 & 0.0204 & 7.63 & 0.15 \\
\hline 10 & 0.1034 & 8.96 & $\underline{0.0422}$ & $\underline{8.58}$ & 0.37 \\
\hline 20 & 0.5356 & 9.28 & $\underline{0.0409}$ & $\underline{8.90}$ & 0.38 \\
\hline 40 & 0.7612 & 9.54 & 0.0135 & 9.41 & 0.13 \\
\hline 80 & 0.8754 & 9.96 & 0.0008 & 9.95 & 0.01 \\
\hline \multicolumn{6}{|c|}{ combined, on/off rate adjustment, $\alpha=1000$} \\
\hline 8 & 0.0301 & 7.75 & 0.0629 & 7.27 & 0.49 \\
\hline 10 & 0.1094 & 8.90 & $\underline{0.1122}$ & $\underline{7.90}$ & 0.99 \\
\hline 20 & 0.5437 & 9.12 & $\underline{0.0658}$ & $\underline{8.52}$ & 0.60 \\
\hline 40 & 0.7706 & 9.17 & 0.0397 & 8.81 & 0.36 \\
\hline 80 & 0.8823 & 9.41 & 0.0199 & 9.22 & 0.19 \\
\hline
\end{tabular}




\section{CONCLUDING REMARKS}

This paper proposes a combined preventive/reactive congestion control approach for highspeed LAN/MAN interconnection networks. An overall control framework which is comprised of four individual control components C1-C4 is described. Furthermore, various implementation strategies and performances of the preventive rate control component $\mathrm{C} 1$ and the reactive feedback control component $\mathrm{C} 3$ are investigated.

The preventive control component $\mathrm{C} 1$ is used primarily to cope with the speedmismatch problem by throttling the long-term average input traffic rate injected into the backbone network under a large propagation delay constraint. The reactive feedback control component $\mathrm{C} 3$, which is aimed at controlling the short-term transient congestion, is designed to complement the preventive control $\mathrm{C} 1$ for additional performance gain under different traffic conditions.

Simulation results demonstrate that the distributed LB rate control algorithm of the preventive control component $\mathrm{C} 1$ effectively limits the aggregate input rate not to exceed the estimated output service rate. It is also shown that additional performance enhancement is attainable by combining the complementary reactive feedback control function C3, although the appropriate choices of implementation policies and/or control parameters seem critically dependent on the traffic characteristics.

As expected, however, the performance enhancement gradually vanishes as the feedback propagation delay increases. In fact, from the control theoretics, no feedback control function can cope with transient congestion where the feedback propagation delay is longer than the transient period.

\section{ACKNOWLEDGEMENT}

This work has been supported by a grant from the ITRC, an Ontario Provincial Centre of Excellence, and the National Science and Engineering Research Council of Canada under grant no. A7779.

\section{REFERENCES}

Sunshine, C.A. (1990) Network interconnection and gateways. IEEE JSAC, 8, 4-11.

Fowler, H.J. and Leland, W.E. (1991) Local area network traffic characteristics, with implications for broadband network congestion management. IEEE JSAC, 9, 11391149 .

Leland, W.E. and Wilson, D.V. (1991) High time-resolution measurement and analysis of LAN traffic: implications for LAN interconnection. IEEE INFOCOM'91, 13601366 .

Jain, R. (1990) Congestion control in computer networks: Issues and trends. IEEE Network, 24-30.

Towsley, D., Fdida, S. and Santoso, H. (1992) Congestion avoidance in high-speed interconnection systems. Computer Networks and ISDN Systems, 24, 185-195. 
Wong, L.N. and Schwartz, M. (1989) Flow control in metropolitan area networks. IEEE INFOCOM'89, 826-833.

Inai, H. and Ohtsuki, K. (1992) Performance study of congestion control for high-speed backbone networks. Computer Communications, 15, 429-437.

Gerla, M., Tai, T.Y. and Gallassi, G. (1992) LAN/MAN interconnection to ATM: A simulation study. IEEE INFOCOM'92, 2270-2279.

Mark, J.W., Lee, B.J. and Mark, B.L. (1994) A combined token/random access protocol for high-speed LAN/MAN ring networks. ICC'94 Conf. Record, 690-695.

IEEE Standard 802.6 (1990) Distributed Queue Dual Bus (DQDB) Metropolitan Area Network (MAN).

Nassehi, M.M. (1990) CRMA: An access scheme for high-speed LANs and MANs. ICC'90 Conf. Record, 1697-1702.

ISO/IEC (1992) Specification of the Asynchronous Transfer Mode Ring (ATMR) Protocol. JTC1/SC6 N7873.

Gerla, M. and Kleinrock, L. (1988) Congestion control in interconnected LANs. IEEE Network, 2, 72-76.

Crocetti, P., Gallassi, G. and Gerla, M. (1991) Bandwidth advertising for MAN/ATM connectionless internetting. IEEE INFOCOM'91, 1145-1150.

Newman, P. (1993) ATM LANs: The customer premises ATM network. 3rd B-ISDN Technical Workshop.

Sidi, M., Liu, W.Z., Cidon, I. and Gopal, I. (1989) Congestion control through input rate regulation. Globecom'89, 1764-1768.

Lee, B.J. and Mark, J.W. (1994) A congestion control scheme in LAN/MAN interconnection. Proc. of CISS'94, 811-815.

Jain, R. and Routhier, S.A. (1986) Packet trains - Measurements and a new model for computer network traffic. IEEE JSAC, 4, 986-995.

B.J. Lee received the B.A.Sc degree from the University of Manitoba, Man., Canada, in 1987, and the M.A.Sc degree from the University of Waterloo, Ont., Canada, in 1989, all in electrical and computer engineering. From 1989 to 1992 he was a Research Associate and has been working towards the Ph.D. degree since 1992 at the university of Waterloo. His research interests include high-speed LAN/MAN protocol, network interconnection and congestion control in broadband networks.

J.W. Mark received the Ph.D. degree in electrical engineering from McMaster University in 1970. He is currently a Professor of Electrical and Computer Engineering at the University of Waterloo, Waterloo, Ontario, Canada. He was an editor of IEEE Trans. on Communications during 1983-1989, and is currently a member of the Intersociety Steering Committee of the IEEE/ACM Trans. on Networking. His research focuses on broadband communication networks, with emphasis on switch design, routing, flow and congestion control, video coding and traffic characterization. 